\title{
Dexterous Estimation of Population Mean in Survey Sampling Under Non-Response Error
}

\author{
S. K. Yadav \\ Department of Statistics, \\ Babasaheb Bhimrao Ambedkar University, Lucknow-226025, India \\ E-mail: drskystats@gmail.com \\ O. P. Yadav \\ Department of Industrial and Manufacturing Engineering, \\ North Dakota State University, Fargo, ND 58104, USA \\ Corresponding author: om.yadav@ndsu.edu \\ D. K. Yadav \\ Department of Commercial Tax, \\ Government of Uttar Pradesh, Lucknow-226010, India \\ Email: dkumar.yadava@gmail.com
}

(Received May 1, 2019; Accepted August 5, 2019)

\begin{abstract}
In this scripture, we ponder the problem of efficient estimation of population mean of study variable utilizing information on highly correlated auxiliary variables under the presence of non-response on either of the variables. For this purpose, we suggest, an improved estimator under three different situations of non-response. Under the first situation, estimation of population mean is done with the problem of non-response on both the study and the auxiliary variables with the additional condition that the population means of the auxiliary variables are known. The second situation is to estimate the population mean of primary variable when the problem of non-response is only on the primary variable but the population means of the auxiliary variables are known while under the third situation estimation is performed with the problem of non-response on both the study and the auxiliary variables but population mean of one of the auxiliary variables is unknown. We study the sampling properties of the suggested estimator under above three different situations of non-response. We compare the proposed estimator with the competing estimators of population mean, under three different situations of non-response. The efficiency conditions are obtained for all three situations. A numerical study is also carried out to verify the efficiency conditions.
\end{abstract}

Keywords- Study variable, Auxiliary variable, Non-response, Bias, MSE, PRE.

\section{Introduction}

Sampling is inevitable whenever the population is large because of the time and money constraints. Further, due to the economic constraint, the mail questionnaire is used to collect the factual information in many sampling surveys. However, in mail questionnaire the problem of non-response is observed at a very large rate and the unknown bias could be a big factor in such situations. On the other hand, the personal interviews generally provide substantially complete response but it is more costly than the mail questionnaire scheme. The purpose of the investigator is to present a scheme which combines the benefits of both the schemes. Generally while combining both the schemes, first the survey questionnaire form is mailed to a large number of target respondents than the required size hopping that total return will be more than expected required size (Hansen and Hurwitz, 1946). 
International Journal of Mathematical, Engineering and Management Sciences

Vol. 4, No. 6, 1307-1324, 2019

https://dx.doi.org/10.33889/IJMEMS.2019.4.6-103

As for as, the estimation of any population parameter is concerned, the most appropriate estimator of any parameter is the corresponding statistic. For example, the most suitable estimator of the population mean is the sample mean of the study variable. Whenever the auxiliary information is used for improved estimation of population mean along with the information on the study variable, it is perceived that the complete factual information on every unit of the sample for both the variables is available and they are correctly measured. But in many surveys, in practice it is very common observation that the problem of non-response occurs in many ways. The nonresponse may be because of unwillingness on the respondent to answer the particular question, non-availability of the respondent, loss of information due to accident or failure by the investigator and missing the observations. The common methodology to deal with the issue of non-response to questionnaire is the personal interview of these non-respondents and collects as much information as possible from them. Many authors contributed to the estimation of population mean of the primary variable using auxiliary information under the presence of nonresponse for different situations of non-responses on study as well as on the auxiliary variables.

El-Badry (1956) suggested a sampling procedure for mailed questionnaires to estimate the population mean of study variable. Rao (1986) along with Khare and Srivastava (1993, 1995, 1997) studied estimation of population mean using ratio type, transformed ratio, product and regression type estimators in presence of non-response. Tracy and Osahan (1994) studied the problem of improved estimation of population mean of main variable under random non-response on study variable versus on study as well as on auxiliary variable.

Singh and Kumar (2008) proposed a regression approach to the estimation of the finite population mean in the presence of non-response. Kumar and Bhougal (2011) suggested elevated estimation of the population mean in presence of non-response. Grover and Kaur (2014) proposed a generalized class of ratio type exponential estimators of population mean under linear transformation of auxiliary variable under the problem of non-response. Bii et al. (2017) suggested estimating a finite population mean under random non-response in survey sampling with replacement scheme. Sharma and Pal (2018) proposed improved ratio type estimator for the estimation of population mean in presence of random non-response. Muneer et al. (2018) suggested a generalized exponential ratio type estimator of population mean in the presence of non-response

Toutenberg and Srivastava (1998); Toutenberg and Srivastava (2003) studied the problem of estimation of ratio of two population means and the population mean respectively in survey sampling when some observations are missing on study and auxiliary variables. Khare and Sinha $(2007,2009)$ also suggested enhanced estimation of the ratio of the two population means using multi auxiliary characteristics in the presence of non-response.

Srinath (1971) estimated the population mean of primary variable under non-response error in multi-phase sampling scheme. Tabasum and Khan (2006); Singh et al. (2010) suggested improved estimation of finite population mean under non response error in two-phase or double sampling scheme. Shabbir and Nasir (2013) also studied estimating the finite population mean using two auxiliary variables in two phase sampling in the presence of non response. Yadav et al. (2018) studied the estimation of finite population mean using known coefficient of variation in the simultaneous presence of non-response and measurement errors under double sampling scheme. 
International Journal of Mathematical, Engineering and Management Sciences

Vol. 4, No. 6, 1307-1324, 2019

https://dx.doi.org/10.33889/IJMEMS.2019.4.6-103

Sud and Srivastva (2000); Srivastava and Shalabh (2001) suggested improved ratio and regression type estimators of population mean under the problem of measurement errors respectively in survey sampling. Kumar et al. (2015); Kumar (2016) studied the problem of estimation of Population Mean in the Presence of Non-Response and Measurement Error both. Singh et al. (2018) studied the effect of measurement error and non-response on estimation of population mean.

The literature indicates continued improvement in the estimators as shown in the form of decreasing mean squared errors (MSE). Lesser MSE represents the sampling distribution of the estimators closer to the true population mean. The purpose of research is to search for the estimator, which can further improve estimation of the true population mean under non-response error. In the present study, we suggest a new estimator of population mean of study variable using known information on the auxiliary variables under the presence of non-response error. Further we study the large sampling properties of the suggested estimator up to the first order of approximation.

The rest of the paper is organized as follows: section two presents the review of existing estimators and proposed estimators are discussed in section 3. Section four represents the proposed estimator whereas the efficiency comparison is discussed in section 5. Section six describes a numerical study and results and discussion are provided in sections 7 and 8 respectively.

\section{Notations}

$N$ : Size of the population under consideration,

$n$ : Size of the sample on which the information is to be taken,

$r$ : Number of units of non-response with the assumption $[r=0,1, \ldots,(n-2)]$,

$Y$ : Study/Main/Primary variable under investigation,

$X$ : Auxiliary/Supplementary/Secondary variable having positive correlation with $Y$,

$Z$ : Auxiliary/Supplementary/Secondary variable having negative correlation with $Y$,

$\bar{Y}, \bar{X}, \bar{Z}$ : Population means of the variable $Y, X$ and $Z$ respectively,

$S_{y}, S_{x}, S_{z}$ : Population standard deviations of the variable $Y, X$ and $Z$ respectively,

$C_{y}, C_{x}, C_{z}$ : Population coefficient of variations of the variable $Y, X$ and $Z$ respectively.

As there are only two possibilities of response or non-response regarding the information on $n$ units of the sample drawn from the population under consideration, therefore the probability distribution of $r$ non-response out of $(n-2)$ possible non-responses along with the $(n-r)$ responses is given by,

$P(r)=\left[\frac{(n-r)}{n-(n-2) p}\right]\left[{ }^{n-2} C_{r} p^{r}(1-p)^{n-2-r}\right]$

where, $p$ is the probability of non-response and it is same (constant) for all possible nonresponses and $r=0,1, \ldots,(n-2)$. 
International Journal of Mathematical, Engineering and Management Sciences

Vol. 4, No. 6, 1307-1324, 2019

https://dx.doi.org/10.33889/IJMEMS.2019.4.6-103

\section{Review of Literature under Different Strategies}

\subsection{Review of Existing Estimators under the First Strategy}

Under this strategy, the non-response is observed on main variable under study and the auxiliary variables while the population mean of the auxiliary variables are known. The following Table 1 represents the competing ratio and product type estimators of population mean of study variable under this strategy by various authors in the literature along with their variances (unbiased estimators) and mean squared errors (biased estimators), up to approximation of order one.

Table 1. Competing estimators under first strategy of non-response along with their MSEs

\begin{tabular}{|c|c|c|c|}
\hline S. No. & Estimators & \multicolumn{2}{|l|}{ Variance/MSE } \\
\hline 1. & $\begin{array}{l}\hat{\bar{Y}}^{*}=\bar{y}^{*} \\
\text { Mean per unit estimator }\end{array}$ & \multicolumn{2}{|l|}{$V\left(\hat{\bar{Y}}^{*}\right)=\lambda^{*} \bar{Y}^{2} C_{y}^{2}$} \\
\hline \multirow[t]{2}{*}{2.} & $\begin{array}{l}\hat{\bar{Y}}_{R}^{*(1)}=\bar{y}^{*}\left(\frac{\bar{X}}{\bar{x}^{*}}\right) \\
\text { Hansen and Hurvitz (1946), Cochran (1977) usual ratio } \\
\text { estimator }\end{array}$ & \multicolumn{2}{|c|}{$\operatorname{MSE}\left(\hat{\bar{Y}}_{R}^{*(1)}\right)=\lambda^{*} \bar{Y}^{2}\left[C_{y}^{2}+C_{x}^{2}\left(1-2 C_{01}\right)\right]$} \\
\hline & $\begin{array}{l}\hat{\bar{Y}}_{P}^{*(1)}=\bar{y}^{*}\left(\frac{\bar{z}^{*}}{\bar{Z}}\right) \\
\text { Khare and Srivastava (1993) usual product estimator }\end{array}$ & \multicolumn{2}{|c|}{$\operatorname{MSE}\left(\hat{\bar{Y}}_{P}^{*(1)}\right)=\lambda^{*} \bar{Y}^{2}\left[C_{y}^{2}+C_{z}^{2}\left(1+2 C_{02}\right)\right]$} \\
\hline \multirow[t]{2}{*}{3.} & $\begin{array}{l}\hat{\bar{Y}}_{\mathrm{Re}}^{*(1)}=\bar{y}^{*} \exp \left(\frac{\bar{X}-\bar{x}^{*}}{\bar{X}+\bar{x}^{*}}\right) \\
\text { Singh and Solanki (2011) ratio type estimator }\end{array}$ & \multicolumn{2}{|c|}{$\operatorname{MSE}\left(\hat{\bar{Y}}_{\mathrm{Re}}^{*(1)}\right)=\lambda^{*} \bar{Y}^{2}\left[C_{y}^{2}+\frac{C_{x}^{2}}{4}\left(1-4 C_{01}\right)\right]$} \\
\hline & $\begin{array}{l}\hat{\bar{Y}}_{P e}^{*(1)}=\bar{y}^{*} \exp \left(\frac{\bar{z}^{*}-\bar{Z}}{\bar{z}^{*}+\bar{Z}}\right) \\
\text { Singh and Solanki (2011) product type estimator }\end{array}$ & \multicolumn{2}{|c|}{$\operatorname{MSE}\left(\hat{\bar{Y}}_{P e}^{*(1)}\right)=\lambda^{*} \bar{Y}^{2}\left[C_{y}^{2}+\frac{C_{z}^{2}}{4}\left(1+4 C_{02}\right)\right]$} \\
\hline 4. & $\begin{array}{l}\hat{\bar{Y}}_{R P e}^{*(1)}=\bar{y}^{*} \exp \left(\frac{\bar{X}-\bar{x}^{*}}{\bar{X}+\bar{x}^{*}}\right) \exp \left(\frac{\bar{z}^{*}-\bar{Z}}{\bar{z}^{*}+\bar{Z}}\right), \\
\text { Sharma and Pal (2018) ratio-cum-product estimator }\end{array}$ & $\operatorname{MSE}\left(\hat{\bar{Y}}_{R P e}^{*(1)}\right)=\lambda^{*} \bar{Y}^{2}$ & $\begin{array}{l}C_{y}^{2}+\frac{1}{4}\left\{C_{x}^{2}\left(1-4 C_{01}\right)\right\} \\
+\frac{1}{4}\left\{C_{z}^{2}\left(1-2 C_{12}+4 C_{02}\right)\right\}\end{array}$ \\
\hline
\end{tabular}

where, $\lambda^{*}=\left(\frac{1}{n q+2 p}-\frac{1}{N}\right), \bar{y}^{*}=\frac{1}{n-r} \sum_{i=1}^{n-r} y_{i}, \quad \bar{x}^{*}=\frac{1}{n-r} \sum_{i=1}^{n-r} x_{i}, \quad \bar{z}^{*}=\frac{1}{n-r} \sum_{i=1}^{n-r} z_{i}$, $C_{01}=\rho_{y x} \frac{C_{y}}{C_{x}}, C_{02}=\rho_{y z} \frac{C_{y}}{C_{z}}$ and $C_{12}=\rho_{x z} \frac{C_{x}}{C_{z}}$.

\subsection{Suggested Estimator under the First Strategy}

Searls (1964) has given a powerful technique to estimate population mean by its corresponding statistic that is sample mean, multiplied with a constant and found the minimum mean squared error of the suggested estimator by finding the optimum value of this constant. Thus motivated by Sharma and Pal (2018); Searls (1964), we suggest the following exponential ratio-cum-product 
International Journal of Mathematical, Engineering and Management Sciences

Vol. 4, No. 6, 1307-1324, 2019

https://dx.doi.org/10.33889/IJMEMS.2019.4.6-103

estimator of population mean of the primary variable, utilizing known information on both the positively and negatively correlated auxiliary variables as,

$t_{R P e}^{*(1)}=\kappa \bar{y}^{*} \exp \left(\frac{\bar{X}-\bar{x}^{*}}{\bar{X}+\bar{x}^{*}}\right) \exp \left(\frac{\bar{z}^{*}-\bar{Z}}{\bar{z}^{*}+\bar{Z}}\right)$

where, $\kappa$ is a suitably chosen constant to be determined such that the MSE of the suggested estimator $t_{R P e}^{*(1)}$ is least.

To study the sampling properties of the suggested estimator, we have the following approximations as,

$\bar{y}^{*}=\bar{Y}\left(1+e_{0}^{*}\right), \bar{x}^{*}=\bar{X}\left(1+e_{1}^{*}\right)$ and $\bar{z}^{*}=\bar{Z}\left(1+e_{2}^{*}\right)$ such that $E\left(e_{0}^{*}\right)=E\left(e_{1}^{*}\right)=E\left(e_{2}^{*}\right)=0$ and $E\left(e_{0}^{* 2}\right)=\lambda^{*} C_{y}^{2}, \quad E\left(e_{1}^{* 2}\right)=\lambda^{*} C_{x}^{2}, \quad E\left(e_{2}^{* 2}\right)=\lambda^{*} C_{z}^{2}, \quad E\left(e_{0}^{*} e_{1}^{*}\right)=\lambda^{*} \rho_{y x} C_{y} C_{x}=\lambda^{*} C_{y x}$, $E\left(e_{0}^{*} e_{2}^{*}\right)=\lambda^{*} \rho_{y z} C_{y} C_{z}=\lambda^{*} C_{y z}, E\left(e_{1}^{*} e_{2}^{*}\right)=\lambda^{*} \rho_{x z} C_{x} C_{z}=\lambda^{*} C_{x z}$.

The proposed estimator $t_{R P e}^{*(1)}$, may be expressed in terms of $e_{i}^{* \prime} s(i=0,1,2)$ as,

$$
\begin{aligned}
t_{R P e}^{*(1)} & =\kappa \bar{Y}\left(1+e_{0}^{*}\right) \exp \left(\frac{\bar{X}-\bar{X}\left(1+e_{1}^{*}\right)}{\bar{X}+\bar{X}\left(1+e_{1}^{*}\right)}\right) \exp \left(\frac{\bar{Z}\left(1+e_{2}^{*}\right)-\bar{Z}}{\bar{Z}\left(1+e_{2}^{*}\right)+\bar{Z}}\right) \\
& =\kappa \bar{Y}\left(1+e_{0}^{*}\right) \exp \left(\frac{-e_{1}^{*}}{2+e_{1}^{*}}\right) \exp \left(\frac{e_{2}^{*}}{2+e_{2}^{*}}\right) .
\end{aligned}
$$

After the expansions and simplifications of the terms on the right hand side of above equation, we have,

$t_{R P e}^{*(1)}=\kappa \bar{Y}\left[1+e_{0}^{*}-\frac{e_{1}^{*}}{2}+\frac{e_{2}^{*}}{2}+\frac{3 e_{1}^{* 2}}{8}-\frac{e_{2}^{* 2}}{8}-\frac{e_{0}^{*} e_{1}^{*}}{2}+\frac{e_{0}^{*} e_{2}^{*}}{2}-\frac{e_{1}^{*} e_{2}^{*}}{4}\right]$

Subtracting $\bar{Y}$ on both sides of equation (2), we have,

$t_{R P e}^{*(1)}-\bar{Y}=\bar{Y}\left[(\kappa-1)+\kappa\left(e_{0}^{*}-\frac{e_{1}^{*}}{2}+\frac{e_{2}^{*}}{2}+\frac{3 e_{1}^{* 2}}{8}-\frac{e_{2}^{* 2}}{8}-\frac{e_{0}^{*} e_{1}^{*}}{2}+\frac{e_{0}^{*} e_{2}^{*}}{2}-\frac{e_{1}^{*} e_{2}^{*}}{4}\right)\right]$

Taking expectations on both sides of (3) and putting values of different expectations, we get the bias of the proposed estimator $t_{R P e}^{*(1)}$ as,

$$
B\left(t_{R P e}^{*(1)}\right)=\bar{Y}\left[(\kappa-1)+\frac{\kappa}{8} \lambda^{*}\left(3 C_{x}^{2}-C_{z}^{2}-4 C_{y x}+4 C_{y z}-2 C_{x z}\right)\right]
$$

Squaring on both sides of (3), taking expectation, simplifying and putting values of different expectations, we get the MSE of the suggested estimator $t_{R P e}^{*(1)}$ as, 
International Journal of Mathematical, Engineering and Management Sciences

Vol. 4, No. 6, 1307-1324, 2019

https://dx.doi.org/10.33889/IJMEMS.2019.4.6-103

$\operatorname{MSE}\left(t_{R P e}^{*(1)}\right)=\bar{Y}^{2}\left[\begin{array}{l}(\kappa-1)^{2}+\frac{\kappa^{2}}{4} \lambda^{*}\left(4 C_{y}^{2}+C_{x}^{2}+C_{z}^{2}-4 C_{y x}+4 C_{y z}-2 C_{x z}\right) \\ +\frac{1}{4}\left(\kappa^{2}-\kappa\right) \lambda^{*}\left(3 C_{x}^{2}-C_{z}^{2}-4 C_{y x}+4 C_{y z}-2 C_{x z}\right)\end{array}\right]$

The $\operatorname{MSE}\left(t_{R P e}^{*(1)}\right)$ is minimum for the optimum value of $\kappa$, given by

$$
\kappa=\frac{\left[1+\frac{\lambda^{*}}{8}\left(3 C_{x}^{2}+C_{z}^{2}-4 C_{y x}+4 C_{y z}-2 C_{x z}\right)\right]}{\left[1+\lambda^{*}\left(C_{y}^{2}+C_{x}^{2}+\frac{C_{z}^{2}}{2}-2 C_{y x}+2 C_{y z}-C_{x z}\right)\right]}=\frac{A}{B}=\kappa_{o p t} \text { (say) }
$$

where,

$$
\begin{aligned}
& A=\left[1+\frac{\lambda^{*}}{8}\left(3 C_{x}^{2}+C_{z}^{2}-4 C_{y x}+4 C_{y z}-2 C_{x z}\right)\right], \text { and } \\
& B=\left[1+\lambda^{*}\left(C_{y}^{2}+C_{x}^{2}+\frac{C_{z}^{2}}{2}-2 C_{y x}+2 C_{y z}-C_{x z}\right)\right] .
\end{aligned}
$$

Putting the optimum value of $\kappa$ from (6) in (5), we get the minimum MSE of the proposed estimator $t_{R P e}^{*(1)}$ as,

$$
M S E_{\text {min }}\left(t_{R P e}^{*(1)}\right)=\bar{Y}^{2}\left[1-\frac{A^{2}}{B}\right]
$$

\subsection{Comparison with Competing Estimators under First Strategy of Non-Response}

\begin{tabular}{|c|c|c|}
\hline S. No. & Estimators & Efficiency condition \\
\hline 1. & $\begin{array}{l}\hat{\bar{Y}}^{*}=\bar{y}^{*} \\
\text { Mean per unit estimator }\end{array}$ & $\begin{array}{l}V\left(\hat{\bar{Y}}^{*}\right)-M S E_{\min }\left(t_{R P e}^{*(1)}\right)>0 \text { or, } \\
\lambda^{*} C_{y}^{2}-\left[1-\frac{A^{2}}{B}\right]>0\end{array}$ \\
\hline 2. & $\begin{array}{l}\hat{\bar{Y}}_{R}^{*(1)}=\bar{y}^{*}\left(\frac{\bar{X}}{\bar{x}^{*}}\right) \\
\text { Hansen and Hurvitz (1946), Cochran (1977) usual ratio } \\
\text { estimator } \\
\hat{\bar{Y}}_{P}^{*(1)}=\bar{y}^{*}\left(\frac{\bar{z}^{*}}{\bar{Z}}\right) \\
\text { Khare and Srivastava (1993) usual product estimator }\end{array}$ & $\begin{array}{l}\operatorname{MSE}\left(\hat{\bar{Y}}_{R}^{*(1)}\right)-M S E_{\min }\left(t_{R P e}^{*(1)}\right)>0 \text { or, } \\
\lambda^{*}\left[C_{y}^{2}+C_{x}^{2}\left(1-2 C_{01}\right)\right]-\left[1-\frac{A^{2}}{B}\right]>0 \\
\operatorname{MSE}\left(\hat{\bar{Y}}_{P}^{*(1)}\right)-M S E_{\min }\left(t_{R P e}^{*(1)}\right)>0 \text { or, } \\
\lambda^{*}\left[C_{y}^{2}+C_{z}^{2}\left(1+2 C_{02}\right)\right]-\left[1-\frac{A^{2}}{B}\right]>0\end{array}$ \\
\hline
\end{tabular}

The following Table 2 represents the efficiency conditions for the suggested estimator to be more efficient than the competing estimators under first strategy of non-response.

Table 2. Comparison with competing estimators under first strategy of non-response 
International Journal of Mathematical, Engineering and Management Sciences

Vol. 4, No. 6, 1307-1324, 2019

https://dx.doi.org/10.33889/IJMEMS.2019.4.6-103

Table 2 continued...

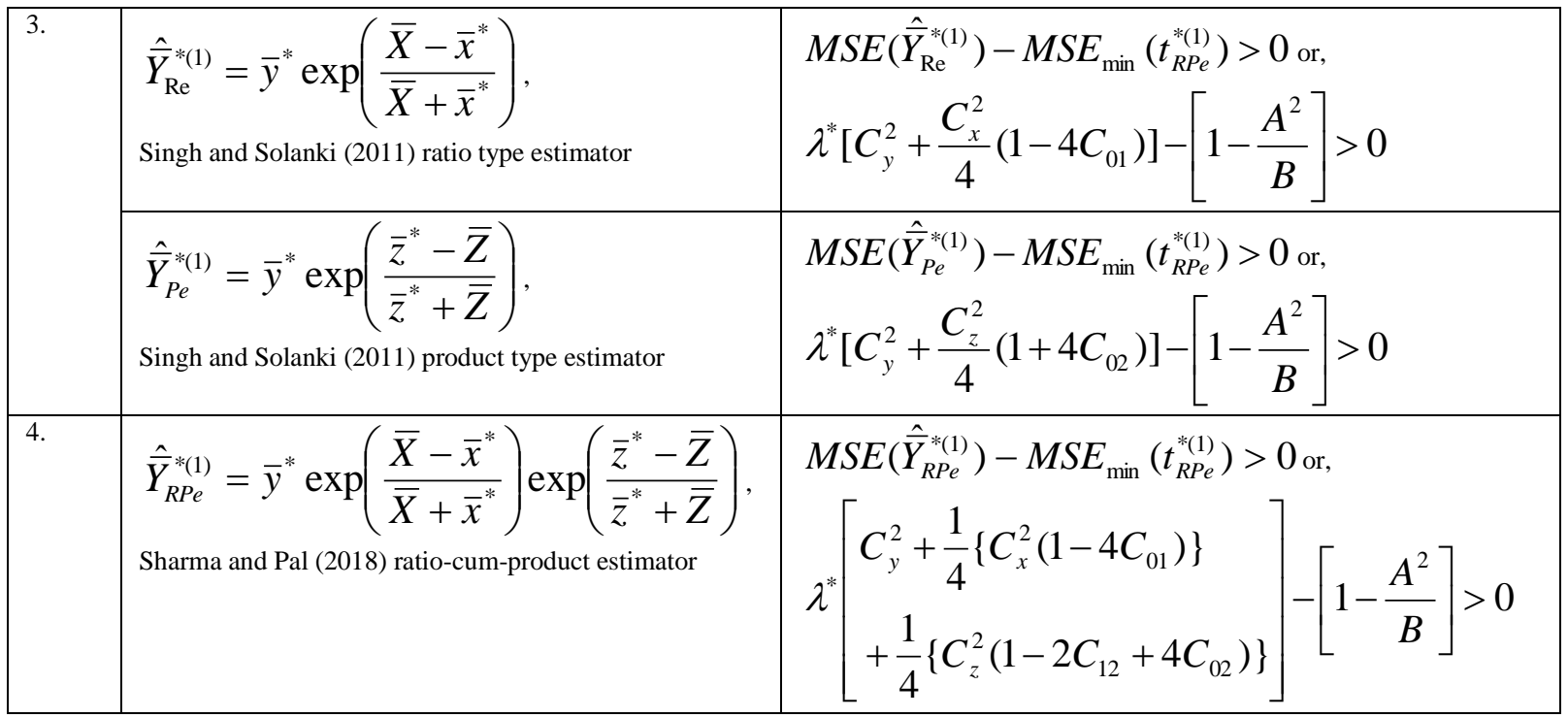

\subsection{Review of Existing Estimators under the Second Strategy}

Under this strategy, the non-response is observed only on the main variable under study while there are complete responses on the auxiliary variables thus the population mean of the auxiliary variables are known. The following Table 3 represents the competing ratio and product type estimators of population mean of study variable under this strategy by various authors in the literature along with their variances (unbiased estimators) and mean squared errors (biased estimators), up to approximation of order one.

Table 3. Competing estimators under second strategy of non-response along with their MSEs

\begin{tabular}{|l|l|l|}
\hline S. No. & Estimators & Variance/MSE \\
\hline 1. & $\begin{array}{l}\hat{\bar{Y}}^{*}=\bar{y}^{*}, \\
\text { Mean per unit estimator }\end{array}$ & $V\left(\hat{\bar{Y}}^{*}\right)=\lambda^{*} \bar{Y}^{2} C_{y}^{2}$ \\
\hline 2. & $\begin{array}{l}\hat{\bar{Y}}_{R}^{*(2)}=\bar{y}^{*}\left(\frac{\bar{X}}{\bar{x}}\right), \\
\text { Rao (1986) usual ratio estimator }\end{array}$ & $\operatorname{MSE}\left(\hat{\bar{Y}}_{R}^{*(2)}\right)=\left[\lambda^{*} \bar{Y}^{2} C_{y}^{2}+\lambda \bar{Y}^{2} C_{x}^{2}\left(1-2 C_{01}\right)\right]$ \\
\cline { 2 - 3 } & $\begin{array}{l}\hat{\bar{Y}}_{P}^{*(2)}=\bar{y}^{*}\left(\frac{\bar{z}}{\bar{Z}}\right), \\
\text { Agrawal and Sthapit (1997) usual product } \\
\text { estimator }\end{array}$ & $\operatorname{MSE}\left(\frac{1}{n}-\frac{1}{N}\right)$ \\
\hline
\end{tabular}


International Journal of Mathematical, Engineering and Management Sciences

Vol. 4, No. 6, 1307-1324, 2019

https://dx.doi.org/10.33889/IJMEMS.2019.4.6-103

Table 3 continued ...

\begin{tabular}{|c|c|c|c|}
\hline \multirow[t]{2}{*}{3.} & $\begin{array}{l}\hat{\bar{Y}}_{\mathrm{Re}}^{*(2)}=\bar{y}^{*} \exp \left(\frac{\bar{X}-\bar{x}}{\bar{X}+\bar{x}}\right) \\
\text { Bahl and Tuteja (1991) type ratio estimator }\end{array}$ & \multicolumn{2}{|c|}{$\operatorname{MSE}\left(\hat{\bar{Y}}_{\mathrm{Re}}^{*(1)}\right)=\left[\lambda^{*} \bar{Y}^{2} C_{y}^{2}+\lambda \bar{Y}^{2} \frac{C_{x}^{2}}{4}\left(1-4 C_{01}\right)\right]$} \\
\hline & $\begin{array}{l}\hat{\bar{Y}}_{P e}^{*(2)}=\bar{y}^{*} \exp \left(\frac{\bar{z}-\bar{Z}}{\bar{z}+\bar{Z}}\right) \\
\text { Bahl and Tuteja (1991) type product estimator }\end{array}$ & \multicolumn{2}{|c|}{$\operatorname{MSE}\left(\hat{\bar{Y}}_{P e}^{*(1)}\right)=\left[\lambda^{*} \bar{Y}^{2} C_{y}^{2}+\lambda \bar{Y}^{2} \frac{C_{z}^{2}}{4}\left(1+4 C_{02}\right)\right]$} \\
\hline 4. & $\begin{array}{l}\hat{\bar{Y}}_{R P e}^{*(2)}=\bar{y}^{*} \exp \left(\frac{\bar{X}-\bar{x}}{\bar{X}+\bar{x}}\right) \exp \left(\frac{\bar{z}-\bar{Z}}{\bar{z}+\bar{Z}}\right) \\
\text { Sharma and Pal (2018) ratio-cum-product } \\
\text { estimator }\end{array}$ & $\operatorname{MSE}\left(\hat{\bar{Y}}_{R P e}^{*(1)}\right)=$ & $\begin{aligned} \lambda^{*} \bar{Y}^{2} C_{y}^{2} & +\frac{1}{4} \lambda \bar{Y}^{2}\left\{C_{x}^{2}\left(1-4 C_{01}\right)\right. \\
& \left.+C_{z}^{2}\left(1-2 C_{12}+4 C_{02}\right)\right\}\end{aligned}$ \\
\hline
\end{tabular}

\subsection{Suggested Estimator under the Second Strategy}

Motivated by Sharma and Pal (2018); Searls (1964), we suggest the following exponential ratiocum-product estimator of population mean of the primary variable, utilizing known information on both the positively and negatively correlated auxiliary variables as,

$t_{R P e}^{*(2)}=\kappa \bar{y}^{*} \exp \left(\frac{\bar{X}-\bar{x}}{\bar{X}+\bar{x}}\right) \exp \left(\frac{\bar{z}-\bar{Z}}{\bar{z}+\bar{Z}}\right)$

where, $\kappa$ is a suitably chosen constant to be determined such that the MSE of the suggested estimator $t_{R P e}^{*(2)}$ is least.

To study the sampling properties of the suggested estimator, we have the following approximations as,

$\bar{y}^{*}=\bar{Y}\left(1+e_{0}^{*}\right), \quad \bar{x}=\bar{X}\left(1+e_{1}\right)$ and $\bar{z}=\bar{Z}\left(1+e_{2}\right)$ such that $E\left(e_{0}^{*}\right)=E\left(e_{1}\right)=E\left(e_{2}\right)=0$ and $E\left(e_{0}^{* 2}\right)=\lambda^{*} C_{y}^{2}, \quad E\left(e_{1}^{2}\right)=\lambda C_{x}^{2}, \quad E\left(e_{2}^{2}\right)=\lambda C_{z}^{2}, \quad E\left(e_{0}^{*} e_{1}\right)=\lambda C_{y x}, \quad E\left(e_{0}^{*} e_{2}\right)=\lambda C_{y z}$, $E\left(e_{1} e_{2}\right)=\lambda C_{x z}$.

The proposed estimator $t_{R P e}^{*(2)}$, may be expressed in terms of $e_{i}^{*} s(i=0,1,2)$ as,

$$
\begin{aligned}
t_{R P e}^{*(2)} & =\kappa \bar{Y}\left(1+e_{0}^{*}\right) \exp \left(\frac{\bar{X}-\bar{X}\left(1+e_{1}\right)}{\bar{X}+\bar{X}\left(1+e_{1}\right)}\right) \exp \left(\frac{\bar{Z}\left(1+e_{2}\right)-\bar{Z}}{\bar{Z}\left(1+e_{2}\right)+\bar{Z}}\right) \\
& =\kappa \bar{Y}\left(1+e_{0}^{*}\right) \exp \left(\frac{-e_{1}}{2+e_{1}}\right) \exp \left(\frac{e_{2}}{2+e_{2}}\right) .
\end{aligned}
$$

After the expansions and simplifications of the terms on the right hand side of above equation, we have,

$$
t_{R P e}^{*(2)}=\kappa \bar{Y}\left[1+e_{0}^{*}-\frac{e_{1}}{2}+\frac{e_{2}}{2}+\frac{3 e_{1}^{2}}{8}+\frac{e_{2}^{2}}{8}-\frac{e_{0}^{*} e_{1}}{2}+\frac{e_{0}^{*} e_{2}}{2}-\frac{e_{1} e_{2}}{4}\right]
$$


International Journal of Mathematical, Engineering and Management Sciences

Vol. 4, No. 6, 1307-1324, 2019

https://dx.doi.org/10.33889/IJMEMS.2019.4.6-103

Subtracting $\bar{Y}$ on both sides of equation (9), we have,

$t_{R P e}^{*(2)}-\bar{Y}=\bar{Y}\left[(\kappa-1)+\kappa\left(e_{0}^{*}-\frac{e_{1}}{2}+\frac{e_{2}}{2}+\frac{3 e_{1}^{2}}{8}+\frac{e_{2}^{2}}{8}-\frac{e_{0}^{*} e_{1}}{2}+\frac{e_{0}^{*} e_{2}}{2}-\frac{e_{1} e_{2}}{4}\right)\right]$

Taking expectations on both sides of (10) and putting values of different expectations, we get the bias of the proposed estimator $t_{R P e}^{*(2)}$ as,

$$
B\left(t_{R P e}^{*(2)}\right)=\bar{Y}\left[(\kappa-1)+\frac{\kappa}{8} \lambda\left(3 C_{x}^{2}+C_{z}^{2}-4 C_{y x}+4 C_{y z}-2 C_{x z}\right)\right]
$$

Squaring on both sides of (10), taking expectation, simplifying and putting values of different expectations, we get the MSE of the suggested estimator $t_{R P e}^{*(2)}$ as,

$$
\operatorname{MSE}\left(t_{R P e}^{*(2)}\right)=\bar{Y}^{2}\left[\begin{array}{l}
(\kappa-1)^{2}+\frac{\kappa^{2}}{4}\left\{4 \lambda^{*} C_{y}^{2}+\lambda\left(C_{x}^{2}+C_{z}^{2}-4 C_{y x}+4 C_{y z}-2 C_{x z}\right)\right\} \\
+\frac{1}{4}\left(\kappa^{2}-\kappa\right) \lambda\left(3 C_{x}^{2}+C_{z}^{2}-4 C_{y x}+4 C_{y z}-2 C_{x z}\right)
\end{array}\right]
$$

The $M S E\left(t_{R P e}^{*(2)}\right)$ is minimum for the optimum value of $\kappa$, given by

$$
\kappa=\frac{\left[1+\frac{\lambda}{8}\left(3 C_{x}^{2}+C_{z}^{2}-4 C_{y x}+4 C_{y z}-2 C_{x z}\right)\right]}{\left[1+\left\{\lambda^{*} C_{y}^{2}+\lambda\left(C_{x}^{2}+\frac{C_{z}^{2}}{2}-2 C_{y x}+2 C_{y z}-C_{x z}\right)\right\}\right]}=\frac{A}{B}=\kappa_{o p t} \text { (say) }
$$

where,

$$
\begin{aligned}
& A=\left[1+\frac{\lambda}{8}\left(3 C_{x}^{2}+C_{z}^{2}-4 C_{y x}+4 C_{y z}-2 C_{x z}\right)\right], \text { and } \\
& B=\left[1+\left\{\lambda^{*} C_{y}^{2}+\lambda\left(C_{x}^{2}+\frac{C_{z}^{2}}{2}-2 C_{y x}+2 C_{y z}-C_{x z}\right)\right\}\right] .
\end{aligned}
$$

Putting the optimum value of $\kappa$ from (13) in (12), we get the minimum MSE of the proposed estimator $t_{R P e}^{*(2)}$ as,

$$
M S E_{\text {min }}\left(t_{R P e}^{*(2)}\right)=\bar{Y}^{2}\left[1-\frac{A^{2}}{B}\right]
$$

\subsection{Comparison with Competing Estimators under Second Strategy of Non- Response}

The following Table 4 represents the efficiency conditions for the suggested estimator to be more efficient than the competing estimators under second strategy of non-response. 
International Journal of Mathematical, Engineering and Management Sciences

Vol. 4, No. 6, 1307-1324, 2019

https://dx.doi.org/10.33889/IJMEMS.2019.4.6-103

Table 4. Comparison with competing estimators under second strategy of non-response

$\left.\begin{array}{|l|l|l|}\hline \text { S. No. } & \text { Estimators } & \text { Efficiency condition } \\ \hline 1 . & \begin{array}{l}\hat{\bar{Y}}^{*}=\bar{y}^{*}, \\ \text { Mean per unit estimator }\end{array} & V\left(\hat{\bar{Y}}^{*}\right)-M S E_{\min }\left(t_{R P e}^{*(1)}\right)>0 \text { or, } \\ & \lambda^{*} C_{y}^{2}-\left[1-\frac{A^{2}}{B}\right]>0\end{array}\right]$

\subsection{Review of Existing Estimators under the Third Strategy}

Under this strategy, the non-response is observed on main variable under study as well as on the auxiliary variables along with the additional condition that the population means of the auxiliary variables are unknown. The following Table 5 represents the competing ratio and product type estimators of population mean of study variable under this strategy by various authors in the literature along with their variances (unbiased estimators) and mean squared errors (biased estimators), up to approximation of order one. 
International Journal of Mathematical, Engineering and Management Sciences

Vol. 4, No. 6, 1307-1324, 2019

https://dx.doi.org/10.33889/IJMEMS.2019.4.6-103

Table 5. Competing estimators under third strategy of non-response along with their MSEs

\begin{tabular}{|c|c|c|}
\hline S. No. & Estimators & Variance/MSE \\
\hline 1. & $\begin{array}{l}\hat{\bar{Y}}^{*}=\bar{y}^{*} \\
\text { Mean per unit estimator }\end{array}$ & $V\left(\hat{\bar{Y}}^{*}\right)=\lambda^{*} \bar{Y}^{2} C_{y}^{2}$ \\
\hline \multirow[t]{2}{*}{2.} & $\begin{array}{l}\hat{\bar{Y}}_{R}^{*(3)}=\bar{y}^{*}\left(\frac{\bar{x}}{\bar{x}^{*}}\right) \\
\text { Rao (1986) usual ratio estimator }\end{array}$ & $\begin{array}{l}\operatorname{MSE}\left(\hat{\bar{Y}}_{R}^{*(3)}\right)=\bar{Y}^{2}\left[\lambda^{*} C_{y}^{2}+\lambda^{\prime} C_{x}^{2}\left(1-2 C_{01}\right)\right] \\
\text { Where, } \lambda^{\prime}=\left(\frac{1}{n q+2 p}-\frac{1}{n}\right)\end{array}$ \\
\hline & $\begin{array}{l}\hat{\bar{Y}}_{P}^{*(3)}=\bar{y}^{*}\left(\frac{\bar{z}^{*}}{\bar{z}}\right) \\
\text { Agrawal and Sthapit (1997) usual product estimator }\end{array}$ & $\operatorname{MSE}\left(\hat{\bar{Y}}_{P}^{*(3)}\right)=\bar{Y}^{2}\left[\lambda^{*} C_{y}^{2}+\lambda C_{z}^{2}\left(1+2 C_{02}\right)\right]$ \\
\hline \multirow[t]{2}{*}{3.} & $\begin{array}{l}\hat{\bar{Y}}_{\mathrm{Re}}^{*(3)}=\bar{y}^{*} \exp \left(\frac{\bar{x}-\bar{x}^{*}}{\bar{x}+\bar{x}^{*}}\right) \\
\text { Singh and Solanki (2011) ratio type estimator }\end{array}$ & $\operatorname{MSE}\left(\hat{\bar{Y}}_{\mathrm{Re}}^{*(3)}\right)=\bar{Y}^{2}\left[\lambda^{*} C_{y}^{2}+\lambda^{\prime} \frac{C_{x}^{2}}{4}\left(1-4 C_{01}\right)\right]$ \\
\hline & $\begin{array}{l}\hat{\bar{Y}}_{P e}^{*(3)}=\bar{y}^{*} \exp \left(\frac{\bar{z}^{*}-\bar{z}}{\bar{z}^{*}+\bar{z}}\right) \\
\text { Singh and Solanki (2011) product type estimator }\end{array}$ & $\operatorname{MSE}\left(\hat{\bar{Y}}_{P e}^{*(3)}\right)=\bar{Y}^{2}\left[\lambda^{*} C_{y}^{2}+\lambda^{\prime} \frac{C_{z}^{2}}{4}\left(1+4 C_{02}\right)\right]$ \\
\hline 4. & $\begin{array}{l}\hat{\bar{Y}}_{R P e}^{*(3)}=\bar{y}^{*} \exp \left(\frac{\bar{x}-\bar{x}^{*}}{\bar{x}+\bar{x}^{*}}\right) \exp \left(\frac{\bar{z}^{*}-\bar{z}}{\bar{z}^{*}+\bar{z}}\right), \\
\text { Sharma and Pal (2018) ratio-cum-product estimator }\end{array}$ & $\operatorname{MSE}\left(\hat{\bar{Y}}_{R P e}^{*(1)}\right)=\bar{Y}^{2}\left[\begin{array}{l}\lambda^{*} C_{y}^{2}+\frac{1}{4} \lambda^{\prime}\left\{C_{x}^{2}\left(1-4 C_{01}\right)\right. \\
+\left(C_{z}^{2}\left(1-2 C_{12}+4 C_{02}\right)\right\}\end{array}\right.$ \\
\hline
\end{tabular}

\subsection{Suggested Estimator under the Third Strategy}

Motivated by Sharma and Pal (2018); Searls (1964), we suggest the following exponential ratiocum-product estimator of population mean of the primary variable, using both the positively and negatively correlated auxiliary variables as,

$t_{R P e}^{*(3)}=\kappa \bar{y}^{*} \exp \left(\frac{\bar{x}-\bar{x}^{*}}{\bar{x}+\bar{x}^{*}}\right) \exp \left(\frac{\bar{z}^{*}-\bar{z}}{\bar{z}^{*}+\bar{z}}\right)$

where, $\kappa$ is a suitably chosen constant to be determined such that the MSE of the suggested estimator $t_{R P e}^{*(3)}$ is least.

To study the sampling properties of the suggested estimator, we have the following approximations as,

$\bar{y}^{*}=\bar{Y}\left(1+e_{0}^{*}\right), \quad \bar{x}^{*}=\bar{X}\left(1+e_{1}^{*}\right), \quad \bar{x}=\bar{X}\left(1+e_{1}\right), \bar{z}^{*}=\bar{Z}\left(1+e_{2}^{*}\right)$ and $\bar{z}=\bar{Z}\left(1+e_{2}\right)$ such that $E\left(e_{0}^{*}\right)=E\left(e_{1}^{*}\right)=E\left(e_{1}\right)=E\left(e_{2}^{*}\right)=E\left(e_{2}\right)=0 \quad$ and $\quad E\left(e_{0}^{* 2}\right)=\lambda^{*} C_{y}^{2}, \quad E\left(e_{1}^{* 2}\right)=\lambda^{*} C_{x}^{2}$, $E\left(e_{1}^{2}\right)=\lambda C_{x}^{2}, \quad E\left(e_{2}^{* 2}\right)=\lambda^{*} C_{z}^{2}, \quad E\left(e_{2}^{2}\right)=\lambda C_{z}^{2}, \quad E\left(e_{0}^{*} e_{1}^{*}\right)=\lambda^{*} C_{y x}, \quad E\left(e_{0}^{*} e_{2}^{*}\right)=\lambda^{*} C_{y z}$, $E\left(e_{1}^{*} e_{2}^{*}\right)=\lambda^{*} C_{x z}, E\left(e_{1}^{*} e_{1}\right)=\lambda^{*} C_{x}^{2}, E\left(e_{2}^{*} e_{2}\right)=\lambda C_{z}^{2}$. 
International Journal of Mathematical, Engineering and Management Sciences

Vol. 4, No. 6, 1307-1324, 2019

https://dx.doi.org/10.33889/IJMEMS.2019.4.6-103

The proposed estimator $t_{R P e}^{*(3)}$, may be expressed in terms of $e_{i}^{* \prime} s(i=0,1,2)$ as,

$$
\begin{aligned}
t_{R P e}^{*(3)} & =\kappa \bar{Y}\left(1+e_{0}^{*}\right) \exp \left(\frac{\bar{X}\left(1+e_{1}\right)-\bar{X}\left(1+e_{1}^{*}\right)}{\bar{X}\left(1+e_{1}\right)+\bar{X}\left(1+e_{1}^{*}\right)}\right) \exp \left(\frac{\bar{Z}\left(1+e_{2}^{*}\right)-\bar{Z}\left(1+e_{2}\right)}{\bar{Z}\left(1+e_{2}^{*}\right)+\bar{Z}\left(1+e_{2}\right)}\right) \\
& =\kappa \bar{Y}\left(1+e_{0}^{*}\right) \exp \left(\frac{e_{1}-e_{1}^{*}}{2+e_{1}+e_{1}^{*}}\right) \exp \left(\frac{e_{2}^{*}-e_{2}}{2+e_{2}^{*}+e_{2}}\right) .
\end{aligned}
$$

After the expansions and simplifications of the terms on the right hand side of above equation, we have,

$$
t_{R P e}^{*(3)}=\kappa \bar{Y}\left[\begin{array}{c}
1+e_{0}^{*}-\frac{\left(e_{1}-e_{1}^{*}\right)}{2}+\frac{\left(e_{2}^{*}-e_{2}\right)}{2}+\frac{\left(e_{0}^{*} e_{1}-e_{0}^{*} e_{1}^{*}\right)}{2}+\frac{\left(e_{0}^{*} e_{2}^{*}-e_{0}^{*} e_{2}\right)}{2} \\
-\frac{e_{1}^{*} e_{2}^{*}}{4}-\frac{3 e_{1}^{* 2}}{8}-\frac{e_{2}^{* 2}}{8}-\frac{e_{1}^{2}}{8}-\frac{e_{1} e_{1}^{*}}{4}+\frac{3 e_{2}^{2}}{8}+\frac{e_{2}^{*} e_{2}}{4}
\end{array}\right]
$$

Subtracting $\bar{Y}$ on both sides of equation (2), we have,

$$
t_{R P e}^{*(3)}-\bar{Y}=\kappa \bar{Y}\left[\begin{array}{c}
1+e_{0}^{*}-\frac{\left(e_{1}-e_{1}^{*}\right)}{2}+\frac{\left(e_{2}^{*}-e_{2}\right)}{2}+\frac{\left(e_{0}^{*} e_{1}-e_{0}^{*} e_{1}^{*}\right)}{2}+\frac{\left(e_{0}^{*} e_{2}^{*}-e_{0}^{*} e_{2}\right)}{2} \\
-\frac{e_{1}^{*} e_{2}^{*}}{4}-\frac{3 e_{1}^{* 2}}{8}-\frac{e_{2}^{* 2}}{8}-\frac{e_{1}^{2}}{8}-\frac{e_{1} e_{1}^{*}}{4}+\frac{3 e_{2}^{2}}{8}+\frac{e_{2}^{*} e_{2}}{4}
\end{array}\right]-\bar{Y}
$$

Taking expectations on both sides of (17) and putting values of different expectations and simplifying, we get the bias of the proposed estimator $t_{R P e}^{*(3)}$ as,

$$
B\left(t_{R P e}^{*(3)}\right)=\bar{Y}\left[(\kappa-1)+\frac{\kappa}{8}\left\{\lambda^{\prime}\left(3 C_{x}^{2}-C_{z}^{2}-4 C_{y x}+4 C_{y z}-2 C_{x z}\right)\right]\right.
$$

Squaring on both sides of (17), taking expectation, simplifying and putting values of different expectations, we get the MSE of the suggested estimator $t_{R P e}^{*(1)}$ as,

$$
\operatorname{MSE}\left(t_{R P e}^{*(3)}\right)=\bar{Y}^{2}\left[\begin{array}{l}
(\kappa-1)^{2}+\frac{\kappa^{2}}{4} \lambda^{\prime}\left(4 C_{y}^{2}+C_{x}^{2}+C_{z}^{2}-4 C_{y x}+4 C_{y z}-2 C_{x z}\right) \\
+\frac{1}{4}\left(\kappa^{2}-\kappa\right) \lambda^{\prime}\left(3 C_{x}^{2}-C_{z}^{2}-4 C_{y x}+4 C_{y z}-2 C_{x z}\right)
\end{array}\right]
$$

The $\operatorname{MSE}\left(t_{R P e}^{*(3)}\right)$ is minimum for the optimum value of $\kappa$, given by

$$
\kappa=\frac{\left[1+\frac{\lambda}{8}\left(3 C_{x}^{2}+C_{z}^{2}-4 C_{y x}+4 C_{y z}-2 C_{x z}\right)\right]}{\left[1+\lambda^{\prime}\left(C_{y}^{2}+C_{x}^{2}+\frac{C_{z}^{2}}{2}-2 C_{y x}+2 C_{y z}-C_{x z}\right)\right]}=\frac{A}{B}=\kappa_{o p t} \text { (say) }
$$


International Journal of Mathematical, Engineering and Management Sciences

Vol. 4, No. 6, 1307-1324, 2019

https://dx.doi.org/10.33889/IJMEMS.2019.4.6-103

where,

$$
\begin{aligned}
& A=\left[1+\frac{\lambda^{\prime}}{8}\left(3 C_{x}^{2}+C_{z}^{2}-4 C_{y x}+4 C_{y z}-2 C_{x z}\right)\right], \text { and } \\
& B=\left[1+\lambda^{\prime}\left(C_{y}^{2}+C_{x}^{2}+\frac{C_{z}^{2}}{2}-2 C_{y x}+2 C_{y z}-C_{x z}\right)\right] .
\end{aligned}
$$

Putting the optimum value of $\kappa$ from (20) in (19), we get the minimum MSE of the proposed estimator $t_{R P e}^{*(3)}$ as,

$M S E_{\min }\left(t_{R P e}^{*(3)}\right)=\bar{Y}^{2}\left[1-\frac{A^{2}}{B}\right]$

\subsection{Comparison with Competing Estimators under Third Strategy of Non-Response}

The following Table 6 represents the efficiency conditions for the suggested estimator to be more efficient than the competing estimators under third strategy of non-response.

\begin{tabular}{|c|c|c|}
\hline S. No. & Estimators & Efficiency condition \\
\hline 1. & $\begin{array}{l}\hat{\bar{Y}}^{*}=\bar{y}^{*} \\
\text { Mean per unit estimator }\end{array}$ & $\begin{array}{l}V\left(\hat{\bar{Y}}^{*}\right)-M S E_{\min }\left(t_{R P e}^{*(3)}\right)>0 \text { or, } \\
\lambda^{*} C_{y}^{2}-\left[1-\frac{A^{2}}{B}\right]>0\end{array}$ \\
\hline \multirow[t]{2}{*}{2.} & $\begin{array}{l}\hat{\bar{Y}}_{R}^{*(3)}=\bar{y}^{*}\left(\frac{\bar{x}}{\bar{x}^{*}}\right) \\
\text { Rao (1986) usual ratio estimator }\end{array}$ & $\begin{array}{l}\operatorname{MSE}\left(\hat{\bar{Y}}_{R}^{*(3)}\right)-M S E_{\min }\left(t_{R P e}^{*(3)}\right)>0 \text { or, } \\
{\left[\lambda^{*} C_{y}^{2}+\lambda^{\prime} C_{x}^{2}\left(1-2 C_{01}\right)\right]-\left[1-\frac{A^{2}}{B}\right]>0}\end{array}$ \\
\hline & $\begin{array}{l}\hat{\bar{Y}}_{P}^{*(3)}=\bar{y}^{*}\left(\frac{\bar{z}^{*}}{\bar{z}}\right) \\
\text { Agrawal and Sthapit (1997) usual product estimator }\end{array}$ & $\begin{array}{l}\operatorname{MSE}\left(\hat{\bar{Y}}_{P}^{*(3)}\right)-M S E_{\min }\left(t_{R P e}^{*(3)}\right)>0 \text { or, } \\
{\left[\lambda^{*} C_{y}^{2}+\lambda^{\prime} C_{z}^{2}\left(1+2 C_{02}\right)\right]-\left[1-\frac{A^{2}}{B}\right]>0}\end{array}$ \\
\hline \multirow[t]{2}{*}{3.} & $\begin{array}{l}\hat{\bar{Y}}_{\mathrm{Re}}^{*(3)}=\bar{y}^{*} \exp \left(\frac{\bar{x}-\bar{x}^{*}}{\bar{x}+\bar{x}^{*}}\right) \\
\text { Singh and Solanki (2011) ratio type estimator }\end{array}$ & $\begin{array}{l}\operatorname{MSE}\left(\hat{\bar{Y}}_{\mathrm{Re}}^{*(3)}\right)-M S E_{\min }\left(t_{R P e}^{*(3)}\right)>0 \text { or, } \\
{\left[\lambda^{*} C_{y}^{2}+\lambda^{\prime} \frac{C_{x}^{2}}{4}\left(1-4 C_{01}\right)\right]-\left[1-\frac{A^{2}}{B}\right]>0}\end{array}$ \\
\hline & $\begin{array}{l}\hat{\bar{Y}}_{P e}^{*(3)}=\bar{y}^{*} \exp \left(\frac{\bar{z}^{*}-\bar{z}}{\bar{z}^{*}+\bar{z}}\right) \\
\text { Singh and Solanki (2011) product type estimator }\end{array}$ & $\begin{array}{l}\operatorname{MSE}\left(\hat{\bar{Y}}_{P e}^{*(3)}\right)-M S E_{\min }\left(t_{R P e}^{*(3)}\right)>0 \text { or, } \\
{\left[\lambda^{*} C_{y}^{2}+\lambda^{\prime} \frac{C_{z}^{2}}{4}\left(1+4 C_{02}\right)\right]-\left[1-\frac{A^{2}}{B}\right]>0}\end{array}$ \\
\hline 4. & $\begin{array}{l}\hat{\bar{Y}}_{R P e}^{*(3)}=\bar{y}^{*} \exp \left(\frac{\bar{x}-\bar{x}^{*}}{\bar{x}+\bar{x}^{*}}\right) \exp \left(\frac{\bar{z}^{*}-\bar{z}}{\bar{z}^{*}+\bar{z}}\right), \\
\text { Sharma and Pal (2018) ratio-cum-product estimator }\end{array}$ & $\begin{array}{l}\operatorname{MSE}\left(\hat{\bar{Y}}_{R P e}^{*(3)}\right)-M S E_{\min }\left(t_{R P e}^{*(3)}\right)>0 \text { or, } \\
{\left[\begin{array}{c}\lambda^{*} C_{y}^{2}+\frac{1}{4} \lambda^{\prime}\left\{C_{x}^{2}\left(1-4 C_{01}\right)\right. \\
+\left(C_{z}^{2}\left(1-2 C_{12}+4 C_{02}\right)\right\}\end{array}\right]-\left[1-\frac{A^{2}}{B}\right]>0}\end{array}$ \\
\hline
\end{tabular}

Table 6. Comparison with competing estimators under third strategy of non-response 
International Journal of Mathematical, Engineering and Management Sciences

Vol. 4, No. 6, 1307-1324, 2019

https://dx.doi.org/10.33889/IJMEMS.2019.4.6-103

\section{Numerical Study}

Under this section, to verify the theoretical efficiency conditions, we have considered the same two natural populations as considered by Sharma and Pal (2018). The first natural population is due to Steel and Torrie (1960, p. 282), where $Y$ represents the log of leaf burn in sec. while $X$ and $Z$ are Potassium percentage and Chlorine percentage respectively. The second natural population is due to Tailor et al. (2014) which depicts $Y$ as the Productivity (MT/Hectare) while $X$ and $Z$ represents Production in '000 Tons and Area in '000 Hectare respectively. The parameters of these populations are given in the following Table 7

Table 7. Parameters of Population-I and Population-II under consideration

\begin{tabular}{|l|l|}
\hline Population-I & Population-II \\
\hline$N=30, n^{\prime}=20, n=6, \bar{Y}=0.6860$, & $N=10, n^{\prime}=6, n=4, \bar{Y}=1.7000$, \\
$\bar{X}=4.6537, \bar{Z}=0.8077, C_{y}=0.4803$, & $\bar{X}=10.4100, \bar{Z}=6.3200, C_{y}=0.2941$, \\
$C_{x}=0.2295, C_{z}=0.7493$, & $C_{x}=0.3391, C_{z}=0.1883$, \\
$\rho_{y x}=0.1794, \rho_{y z}=-0.4996$, & $\rho_{y x}=0.9065, \rho_{y z}=-0.0840$, \\
$\rho_{x z}=0.4074$ & $\rho_{x z}=0.3286$ \\
\hline
\end{tabular}

The following Table 8 and Table 9 represents the percentage relative efficiency (PRE) of various competing estimators of population mean along with the suggested estimator with respect to the mean per unit estimator under the problem of non-response error under three different strategies.

Table 8. PRE of various estimators with respect to the mean per unit estimator for the Population-I

\begin{tabular}{|l|c|c|c|c|}
\hline S. No. & Estimator & PRE under Strategy-I & PRE under Strategy-II & PRE under Strategy-III \\
\hline 1. & $\hat{\bar{Y}}^{*}$ & 100.00 & 100.00 & 100.00 \\
\hline 2. & $\hat{\bar{Y}}_{R}^{*}$ & 094.62 & 095.73 & 98.79 \\
\hline 3. & $\hat{\bar{Y}}_{P}^{*}$ & 053.33 & 059.29 & 84.79 \\
\hline 4. & $\hat{\bar{Y}}_{\mathrm{Re}}^{*}$ & 102.95 & 102.29 & 100.62 \\
\hline 5. & $\hat{\bar{Y}}_{P e}^{*}$ & 120.62 & 115.45 & 103.82 \\
\hline 6. & $\hat{\bar{Y}}_{R P e}^{*}$ & 154.18 & 138.07 & 108.18 \\
\hline 7. & $t_{R P e}^{*}$ & 156.89 & 142.56 & 111.84 \\
\hline
\end{tabular}


International Journal of Mathematical, Engineering and Management Sciences

Vol. 4, No. 6, 1307-1324, 2019

https://dx.doi.org/10.33889/IJMEMS.2019.4.6-103

Table 9. PRE of various estimators with respect to the mean per unit estimator for the Population-II

\begin{tabular}{|c|c|c|c|c|}
\hline S. No. & Estimator & PRE under Strategy-I & PRE under Strategy-II & PRE under Strategy-III \\
\hline 1. & $\hat{\bar{Y}}^{*}$ & 100.00 & 100.00 & 100.00 \\
\hline 2. & $\hat{\bar{Y}}_{R}^{*}$ & 418.49 & 252.49 & 118.63 \\
\hline 3. & $\hat{\bar{Y}}_{P}^{*}$ & 076.79 & 080.65 & 094.12 \\
\hline 4. & $\hat{\bar{Y}}_{\mathrm{Re}}^{*}$ & 348.23 & 230.25 & 117.25 \\
\hline 5. & $\hat{\bar{Y}}_{P e}^{*}$ & 095.35 & 096.28 & 099.00 \\
\hline 6. & $\hat{\bar{Y}}_{R P e}^{*}$ & 466.09 & 265.45 & 119.34 \\
\hline 7. & $t_{R P e}^{*}$ & 478.72 & 276.95 & 128.38 \\
& (Proposed) & & & \\
\hline
\end{tabular}

The PRE of the competing and the suggested estimators are shown in Figure 1 and Figure 2 for all three sampling strategies of non-response.

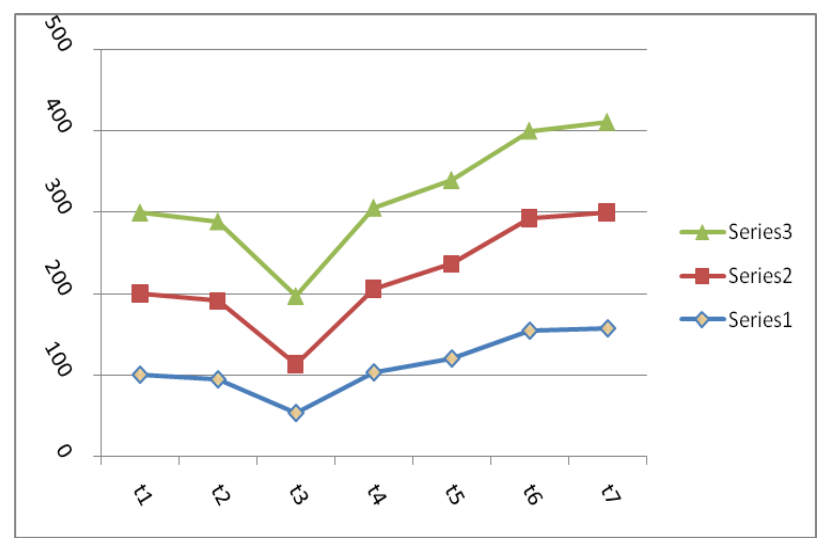

Figure 1. PRE of estimators for Population-1

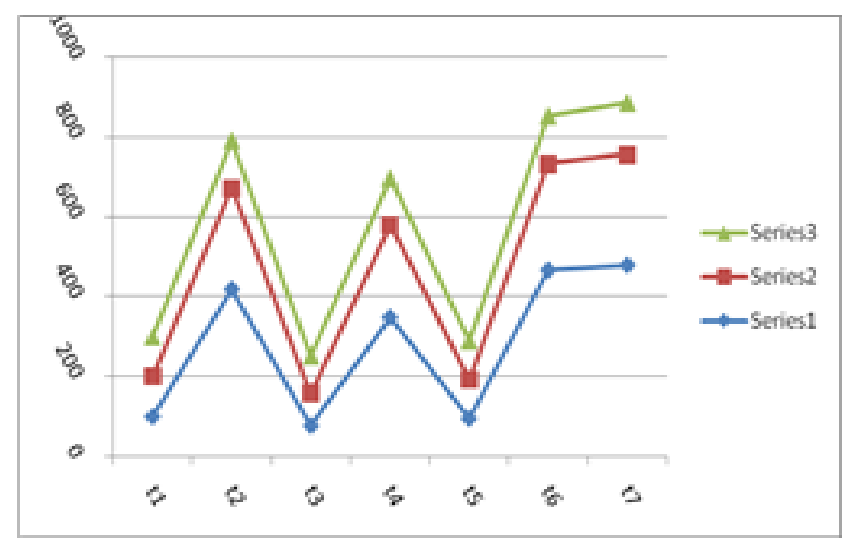

Figure 2. PRE of estimators for Population-2 
International Journal of Mathematical, Engineering and Management Sciences

Vol. 4, No. 6, 1307-1324, 2019

https://dx.doi.org/10.33889/IJMEMS.2019.4.6-103

\section{Results and Discussion}

From Table 8, it can be seen that the PREs of various competing estimators for the Population-I with respect to the mean per unit estimator for all three strategies lie between [053.33 154.18], [059.29 138.07] and [84.79 108.18] while the PREs of the suggested estimators for these three strategies are 156.89, 142.56 and 111.84 respectively, which are more than all the other competing estimators. Similarly, it is evident from the PREs of various competing estimators for the Population-II with respect to the mean per unit estimator for three strategies lie between [076.79 466.09], [080.65 265.45] and [094.12 119.34] while the PREs of the suggested estimators for these three strategies are 478.72, 276.95 and 128.38 respectively representing more efficient with respect to the mean per unit estimator, fulfilling the purpose of the research.

\section{Conclusion}

In the present scripture, we suggested a new exponential ratio-cum-product type estimator for elevated estimation of population mean of the study variable utilizing both positively and negatively correlated auxiliary information under the problem of non-response for three different situations of non-response. We studied the bias and mean squared errors of the suggested estimator up to the approximation of degree one using Taylor's series expansion. The suggested estimator is compared theoretically with the competing estimators under three different strategies of non-response and the conditions for the suggested estimator to be more efficient than the competing estimators are obtained. These efficiency conditions are verified using two natural populations and it have been shown in Table 8 and Table 9 that the suggested estimator is more efficient than other mentioned competing estimators of population mean under the problem of non-response. Thus the suggested estimator may be applied for improved estimation of population mean under the situation of non-response.

\section{Conflict of Interest}

The authors have no conflict of interest regarding the publication of the article.

\section{Acknowledgment}

The authors sincerely thank anonymous reviewers and the Editor-in-Chief for providing critical comments and suggestions for improving the quality of the paper.

\section{References}

Agrawal, M.C., \& Sthapit, A.B. (1997). Hierarchic predictive ratio-based and product-based estimators and their efficiency. Journal of Applied Statistics, 24(1), 97-104.

Bahl, S., \& Tuteja, R. (1991). Ratio and product type exponential estimators. Journal of Information and Optimization Sciences, 12(1), 159-164.

Bii, N.K., Onyango, C.O., \& Odhiambo, J. (2018, November). Estimating a finite population mean under random non response in two stage cluster sampling with replacement. In Journal of Physics: Conference Series, 1132(1), p. 012071. IOP Publishing.

Cochran, W.G. (1977). Sampling techniques. New-York, John Wiley and Sons. 
International Journal of Mathematical, Engineering and Management Sciences

Vol. 4, No. 6, 1307-1324, 2019

https://dx.doi.org/10.33889/IJMEMS.2019.4.6-103

El-Badry, M.A. (1956). A sampling procedure for mailed questionnaires. Journal of the American Statistical Association, 51(274), 209-227.

Grover, L.K., \& Kaur, P. (2014). A generalized class of ratio type exponential estimators of population mean under linear transformation of auxiliary variable. Communications in Statistics-Simulation and Computation, 43(7), 1552-1574.

Hansen, M.H., \& Hurwitz, W.N. (1946). The problem of non-response in sample surveys. Journal of the American Statistical Association, 41(236), 517-529.

Khare, B.B., \& Sinha, R.R. (2007). Estimation of the ratio of the two population means using multi auxiliary characteristics in the presence of non-response. In Statistical Techniques in Life Testing, Reliability, Sampling Theory and Quality Control. 1, 63-171.

Khare, B.B., \& Sinha, R.R. (2009). On the class of estimators for population mean using multi auxiliary characters in the presence of non-response. Statistics in Transition-New Series, 10(1), 3-14.

Khare, B.B., \& Srivastava, S. (1993). Estimation of population mean using auxiliary character in presence of non-response. National Academy Science Letters, 16, 111-111.

Khare, B.B., \& Srivastava, S. (1995). Study of conventional and alternative two phase sampling ratio, product and regression estimators in presence of nonresponse. Proceedings-National Academy Of Sciences India Section A, 65, 195-204.

Khare, B.B., \& Srivastava, S. (1997). Transformed ratio type estimators for the population mean in the presence of nonresponse. Communications in Statistics-Theory and Methods, 26(7), 1779-1791.

Kumar S. (2016). Improved estimation of population mean in presence of nonresponse and measurement error. Journal of Statistical Theory and Practice, 10 (4), 707-720.

Kumar, S., \& Bhougal, S. (2011). Estimation of the population mean in presence of nonresponse. Communications for Statistical Applications and Methods, 18(4), 537-548.

Kumar, S., Bhougal, S., Nataraja, N.S., \& Viswanathaiah, M. (2015). Estimation of population mean in the presence of non-response and measurement error. Revista Colombiana de EstadÝstica, 38(1), 145-161.

Muneer, S., Shabbir, J., \& Khalil, A. (2018). A generalized exponential type estimator of population mean in the presence of non-response. Statistics in Transition-New Series, 19(2), 259-276.

Rao, P.S.R.S. (1986). Ratio estimation with sub sampling the non-respondents. Survey Methodology, 12(2), 217-230.

Searls, D.T. (1964). The utilization of a known coefficient of variation in the estimation procedure. Journal of the American Statistical Association, 59(308), 1225-1226.

Shabbir, J., \& Khan, N.S. (2013). On estimating the finite population mean using two auxiliary variables in two phase sampling in the presence of non response. Communications in Statistics-Theory and Methods, 42(22), 4127-4145.

Sharma, P., \& Pal, S.K. (2018). Estimation of population mean in presence of random non-response. Journal of Statistics and Management Systems, 21(6), 1105-1119.

Singh, H.P., \& Kumar, S. (2008). A regression approach to the estimation of the finite population mean in the presence of non-response. Australian \& New Zealand Journal of Statistics, 50(4), 395-408.

Singh, H.P., \& Solanki, R.S. (2011). Estimation of finite population mean using random non-response in survey sampling. Pakistan Journal of Statistics and Operations Research, 7(1), 21-41.

Singh, H.P., Kumar, S., \& Kozak, M., (2010) Improved estimation of finite population mean using subsampling to deal with non response in two phase sampling scheme. Communication in StatisticsTheory and Method, 39(5), 791-802. 
International Journal of Mathematical, Engineering and Management Sciences

Vol. 4, No. 6, 1307-1324, 2019

https://dx.doi.org/10.33889/IJMEMS.2019.4.6-103

Singh, P., Singh, R., \& Bouza, C.N. (2018). Effect of measurement error and non-response on estimation of population mean. Revista Investigacion Operacional, 39(1), 108-120.

Srinath, K.P. (1971). Multiphase sampling in non-response problems. Journal of American Statistical Association, 66(335), 583-586.

Srivastava, A.K., \& Shalabh (2001). Effect of measurement errors on the regression method of estimation in survey sampling. Journal of Statistical Research, 35(2), 35-44.

Steel, R.G.D., \& Torrie, J.H. (1960). Principles and procedures of statistics. McGraw-Hill Book Company, Inc., New York, Toronto, London.

Sud, U.C., \& Srivastava, S.K. (2000). Estimation of population mean in repeat surveys in the presence of measurement errors. Journal of Indian Society of Agricultural Statistics, 53(2), 125-133.

Tabasum, R., \& Khan, I.A. (2006). Double sampling for ratio estimator for the population mean in the presence of non-response. Assam Statistical Review, 20, 73-83.

Tailor, R., Chouhan, S., \& Kim, J.M. (2014). Ratio and product type exponential estimators of population mean in double sampling for stratification. Communications for Statistical Applications and Methods, 21(1), 1-9.

Toutenburg, H., \& Srivastava, V.K. (1998). Estimation of ratio of population means in survey sampling when some observations are missing. Metrika, 48(3), 177-187.

Toutenburg, H., \& Srivastava, V.K. (2003). Efficient estimation of population mean using incomplete survey data on study and auxiliary characteristics. Statistica, 63(2), 223-236.

Tracy, D.S., \& Osahan, S.S. (1994). Random non-response on study variable versus on study as well as auxiliary variables. Statistica, 54(2), 163-168.

Yadav, D.K., Devi, M., \& Yadav, S.K. (2018). Estimation of finite population mean using known coefficient of variation in the simultaneous presence of non-response and measurement errors under double sampling scheme. Journal of Reliability and Statistical Studies, 11(1), 51-66. 